\title{
Research on Effective Information Visualization Method Based on Mobile Web
}

\author{
Jina YOO ${ }^{1}$, Tae-Hyeong KIM ${ }^{2}$
}

Received: May 23, 2020. Revised: July 02, 2020. Accepted: July 05, 2020

\begin{abstract}
Purpose: The purpose of this study is to recognize the role and necessity of public data visualization through prior research, investigation, and data verification processes. In addition, this study intends to check what factors should be considered in order to visualize data on the mobile web. Through this process, by identifying the cognitive load affecting information visualization by type, as a result, I would like to propose an effective information visualization method to effectively deliver public data related to government policies. Research design, data and methodology: In this study, we analyzed the case of information visualization according to infographics, which has been widely used in the public field among various visualization methods. For this study, a questionnaire survey was conducted for young people in their 20s and 30s with the highest mobile usage rate. Results: Based on the results, IPA (Importance Performance Analysis) was performed to conduct cognitive load test tools for information visualization of public data and confirmed the implications for each type of infographics. Conclusions: As a result of research, in order to efficiently deliver public data on the mobile web, first, it is necessary to construct a visual screen that can be easily identified through clear data. Appropriate graphic elements can be used according to the type to make it easier for users to acquire and understand information. Second, it is necessary to provide useful content in visualizing information. Third, in order to efficiently transmit information and increase understanding of data, it is necessary to visualize information that can induce interest in data and form metaphors. Fourth, it is necessary to visualize information to reduce cognitive load in terms of physical and mental aspects in order to accommodate users' comfortable information. Fifth, in order to effectively deliver public data, it is necessary to compose contents and information that are easy for users to understand. This study examines effective information visualization methods to increase the communication effect of public data in response to changes in the data-based intelligent information society and suggests implications for each type considering cognitive loads to help future public institutions to communicate and accept information.
\end{abstract}

Keywords : Information visualization, Public information, IPA, Cognitive load theory.

JEL Classification Code : E44, F31, F37, G15

\section{1. 서론}

1 First Author. Master's degree graduate, Department of Data Knowledge Service Engineering, Dankook University, Korea. Email: ussun@nate.com

2 Corresponding Author, Professor, Department of Data Knowledge Service Engineering, Dankook University, Korea, Email: kimtoja@dankook.ac.kr

(c) Copyright: The Author(s)

This is an Open Access article distributed under the terms of the Creative Commons Attribution Non-Commercial License (https://creativecommons.org/licenses/by-nc/4.0/) which permits unrestricted non-commercial use, distribution, and reproduction in any medium, provided the original work is properly cited.
데이터가 폭발적으로 증가하고 있는 환경에서 수많은 정보와 스마트폰 사용자가 급증하며 스마트폰을 통해 필요한 정보를 찾는 사용자들이 점점 증가하고 있다. 과학기술정보통신부의 자료에 따르면 2018 년도 스마트폰 사용 비중은 94.3\%로 매우 높게 나타났다. 사용자들이 증가함에 따라 정부 부처에서도 정책 또는 관련 데이터들의 정보를 시각화하여 모바일웹 환경을 통해 사용자에게 전달하는 추세이다. 정부 부처의 업무 수행 및 홍보활동 중 정책 홍보활동에 대한 조사결과에 따르면 정부 정책 소식이나 홍보물 접촉 경로는 스마트폰이 $60 \%$ 로 가장 높고 TV $55 \%$, 컴퓨터 $42 \%$ 순으로 나타났다(LipSourceKorea, 2019). 
2009 년 아이폰이 국내에 첫 등장한 후, 만 6 세 이상 인구의 $910 \%$ 가스마트폰'을 보유하는 등 스마트 기기의 보유율은 급속히 증가하였다. 정보의 습득이 매우 중요했던 과거와 다르게 정보의 접근성이 매우 용이해진 현 시대에서는 개인이 직접 정보의 선별을 진행한다. 특히 요즘과 같이 빠르게 변화되는 시대에는 넘쳐나는 정보와 사용자들의 커뮤니케이션 방법에 관해 근본적인 패러다임의 전환이 필요하며 이에 정보와 수용자 사이에 상호작용을 원활하게 해주는 정보의 시각화가 중요해졌다(Choi \& Kim, 2012, Yoo, Suh, \& Kim, 2020). 최근에는 공공데이터와 다양한 빅데이터가 개방되면서 공개된 데이터를 활용하여 의사결정을 한다KKim, Park, \& Suh, 2019). 정부는 다양한 데이터 사업을 추진하여 공공데이터 활용을 적극 지원하고 있으며 이러한 추세는 점차 가속화될 전망이다(Lee \& Lee, 2020). 하지만 공개된 공공데이터는 크기가 방대하고 다양한 데이터 종류와 항목을 포함하고 있다. 따라서 데이터에 대한 지식이 없는 일반인은 이러한 데이터를 사용하고 데이터의 의미를 파악하는 것에 한계가 있다. 이러한 흐름에 따라 수용자가 쉽게 이해하고 관심과 흥미를 높이고 공감을 불러일으킬 수 있는 시각화의 한 방법으로써 인포그래픽스(infographicss)이 주목받고 있다(Lee, 2017). 특히 인포그래픽스는 데이터 시각화와 달리 홍보나 정보공개 차원의 분석보다는 신문이나 공공, 연구원을 중심으로 자료를 전달하는 영역을 크게 점유하고 있다는 점에서 향후 활용에의 차별성을 가질 것으로 기대한다(Ahn, 2016).

이에 본 연구는 공공 데이터에 있어 모바일웹 기반에서 보다 효과적인 정보시각화 방안을 제안하고자 하며, 특히 사용자의 인지부하를 고려하여 세부적으로 인포그래픽스라는 방법을 통해 효과적인 정보시각화 방안을 제시한다. 최근 정부 및 공공기관에서 제시하는 정책 관련 공공데이터의 경우 인포그래픽스를 통해 정보전달을 하는 경우가 많은 바, 데이터 기반의 지능정보사회로의 변화에 따라 공공데이터의 커뮤니케이션 효과를 높이기 위한 효과적인 정보시각화 방식을 살펴보고, 인지부하를 고려한 유형별 시사점을 제시함으로써 향후 공공기관의 정보전달 및 수용에 도움을 주고자 한다.

\section{2. 선행연구 고찰}

\section{1. 모바일 웹(Mobile web)}

모바일웹은 웹 기술로 개발되어 스마트폰 브라우저에서 실행되는 개념으로, 무선 인터넷 및 스마트폰의 등장과 더불어 시간, 장소에 상관없이 스마트폰 사용자들의 정보 이용이 늘어나면서 그 수요가 폭발적으로 증가하였다. 특히 웹이라는 동일한 플랫폼을 사용하여
PC(Personal computer) 웹사이트에서 사용하던 콘텐츠의 재활용 및 확장이 가능해짐으로써 모바일웹은 주로 Script 언어, HTML, CSS 등을 이용하여 특정 서버 내에 구현하는 방식으로써 일반적으로 웹브라우저에서 접근하므로 앱과 달리 특별한 설치를 요하지 않는다 (Ahn, 2016; Cho, Kim, \& Ryoo, 2011). 따라서 사용자 관점에서는 다운로드 또는 설치라는 추가적인 작업이 필요 없으며, 개발자 관점에서는 스마트폰 모델에 따라 추가적인 개발을 할 필요가 없어 편하다는 장점이 있다(Song, 2011). 그러나 작은 화면과 한 눈에 보기 힘든 구조적 문제 및 정보를 제공받기 위해 소요되는 시간 등 사용상 요구되어지는 부분이 PC와 다르다. 따라서 웹사이트에 비해 정보의 구성이 단순하고, 접근과 사용성이 용이해야 하므로 보다 효과적인 정보 전달을 위해 새로운 모델 또는 방식이 요구된다(Choi, Kim, \& Kim, 2014).

\section{2. 정보시각화}

정보시각화란 사용자에게 더 효과적으로 정보 전달을 위하여 그래픽 요소를 사용하여 데이터가 정보로서 의미가 생성되도록 형상화되는 것을 뜻한다(Kim, 2018).

Table 1: Type of infographics

\begin{tabular}{|c|l|}
\hline Type & \multicolumn{1}{c|}{ Characteristics } \\
\hline Map & $\begin{array}{l}\text { A format that contains information in a map } \\
\text { of a specific country or region } \\
\text { Easy to display various statistical data by } \\
\text { country and region }\end{array}$ \\
\hline Graphic & $\begin{array}{l}\text { Ways to store information using various } \\
\text { tables and graphs } \\
\text { Represent all kinds of resin data }\end{array}$ \\
\hline Timeline & $\begin{array}{l}\text { A way to show the history or development } \\
\text { related to the subject in the form of a timeline } \\
\text { Easy to communicate information related } \\
\text { to history, origin, evolution, etc. }\end{array}$ \\
\hline Storytelling & $\begin{array}{l}\text { Organized to tell a story about an event or } \\
\text { subject } \\
\text { Deliver all aspects of a specific event in all } \\
\text { directions }\end{array}$ \\
\hline Animation & $\begin{array}{l}\text { Applicability data related to daily life can be } \\
\text { applied using cartoon elements such as } \\
\text { characters } \\
\text { Easy to express information related to } \\
\text { behavior, occupation, psychology, etc. }\end{array}$ \\
\hline analysis & $\begin{array}{l}\text { Compare two or more types or concepts of } \\
\text { information } \\
\text { Compare various information and concept } \\
\text { characteristics of a specific category at a } \\
\text { glance }\end{array}$ \\
\hline
\end{tabular}


정보시각화는 정해진 공간에 많은 데이터를 효과적으로 또는 차별적으로, 그리고 정보에 대한 이야기를 창출할 때 유용하다. 정보시각화는 프랑스의 지도학자이자 정보시각화 분야의 전문가인 Jacques Bertin 과 데이터 그래픽 분야의 거장인 Edward Tufte 를 비롯하여 1990 년대에 이후 전문연구 분야로서 꾸준히 발전해왔다. 최근엔 데이터 시각화(Data Visualization), 비주얼 애널리틱스(Visual Analytics), 인포그래픽스 등으로 세분화되고 있다. 데이터시각화는 다량의 데이터들을 압축하여 주로 그래프의 형식으로 시각화하는 것이며, 비주얼 애널리틱스는 대화식 인터페이스로 촉진되는 분석 추론 기술로써 정보의 상호작용을 기반으로 한다. 인포그래픽스는 데이터시각화와는 구별될 수 있을 정도로 원(Raw) 데이터는 취급하지 않으며 가장 실용적으로 전달하기 위한 방향에서 차트, 다이어그램, 일러스트레이션 등이 다양하게 사용되어 진다(Kim, 2018). 인포그래픽스의 그래픽들은 지도, 저널리즘, 기술적 산출물, 교육 결과 등과 같은 복잡한 정보를 빠르고 명확하게 표현하기 위해 발전되어 온 분야로써, 다음과 같은 유형으로 분류할 수 있다(Lee, 2013).

\section{3. 인지부하}

Table 2: Cognitive load measurement summary table (Ryu \& Lim, 2009)

\begin{tabular}{|c|c|c|c|c|c|}
\hline Type & $\begin{array}{c}\text { Physicl } \\
\text { exertion }\end{array}$ & $\begin{array}{c}\text { Mentl } \\
\text { effort }\end{array}$ & $\begin{array}{c}\text { Difficuly } \\
\text { of the } \\
\text { task }\end{array}$ & $\begin{array}{c}\text { Selfeval } \\
\text { uation }\end{array}$ & $\begin{array}{c}\text { Data } \\
\text { design }\end{array}$ \\
\hline $\begin{array}{c}\text { Tribble } \\
(2001)\end{array}$ & & $\bullet$ & $\bullet$ & & \\
\hline $\begin{array}{c}\text { Adcock } \\
\text { (2004) }\end{array}$ & $\bullet$ & $\bullet$ & $\bullet$ & & \\
\hline Kim (2004) & $\bullet$ & $\bullet$ & $\bullet$ & & \\
\hline \begin{tabular}{c} 
Lee (2004) \\
\hline $\begin{array}{c}\text { Wilkinson } \\
(2004)\end{array}$
\end{tabular} & $\bullet$ & $\bullet$ & $\bullet$ & $\bullet$ & \\
\hline $\begin{array}{c}\text { Christensen } \\
(2005)\end{array}$ & & $\bullet$ & $\bullet$ & & \\
\hline $\begin{array}{c}\text { Whelan } \\
(2006)\end{array}$ & $\bullet$ & $\bullet$ & $\bullet$ & $\bullet$ & $\bullet$ \\
\hline $\begin{array}{c}\text { Burkes } \\
(2007)\end{array}$ & & $\bullet$ & $\bullet$ & & \\
\hline Miller (2007) & $\bullet$ & $\bullet$ & $\bullet$ & & \\
\hline $\begin{array}{c}\text { Hancock- } \\
\text { Niemic } \\
(2008)\end{array}$ & $\bullet$ & $\bullet$ & $\bullet$ & $\bullet$ & \\
\hline
\end{tabular}

인지부하는 학습 또는 해결해야 하는 문제 수행 과정에서의 인지적 요구량을 의미하는 것으로, 주로 효과적인 학습결과를 촉진하기 위한 방향에서 주로 활용된다. 이를 측정하기 위해서는 주로 주관적 방식의
설문지법, 반응 시간 측정법 또는 생리신호 측정법 등의 방법을 사용한다(Ryu \& Yim, 2009). 일반적으로 가장 많이 활용하는 방법은 첫 번째, 사용자의 주관적 판단을 중심으로 설문하는 것이다. 두 번째는 인지반응에 대한 시간을 측정하는 것이다. 인지반응의 측정 시간은 주로 이중 과제 분석(Dual-task Analysis)을 활용한다. 이는 일차 과제(Primary Task), 이차 과제(Secondary Task)를 나란히 제시한 다음 과제 수행에 따른 반응 시간을 측정하여 인지부하의 정도를 측정하는 것으로, 이차 과제에 대한 반응이 빠를수록 인지부하의 정도가 낮다(Ryu \& Yim, 2009). 세 번째로 생리신호를 측정하여 인지부하를 확인하는 방법이다. kehara and Crosby(2005)는 생리신호의 사용은 인지부하를 측정하는 정확한 방법 중 하나라고 하였으며, 특히 실시간으로 정보의 수집이 가능하다는 장점이 있다.

\section{3. 연구 방법론}

\section{1. 조사 대상 선정}

Table 3: General characteristics of survey subjects

\begin{tabular}{|c|c|c|c|}
\hline Type & Item & Frequency & Percentage \\
\hline \multirow{3}{*}{ Sex } & Male & 46 & 51.69 \\
\hline & Female & 43 & 48.31 \\
\hline & Sum & 89 & 100 \\
\hline \multirow{5}{*}{ Age } & $20 \sim 25$ & 29 & 32.58 \\
\hline & $25 \sim 30$ & 26 & 29.21 \\
\hline & $30 \sim 35$ & 19 & 2135 \\
\hline & $35 \sim 40$ & 15 & 16.85 \\
\hline & Sum & 89 & 100 \\
\hline \multirow{5}{*}{$\begin{array}{l}\text { Public data } \\
\text { Infographics } \\
\text { Main path of } \\
\text { contact }\end{array}$} & $\begin{array}{l}\text { Government/Public } \\
\text { Institution Website }\end{array}$ & 16 & 17.98 \\
\hline & $\begin{array}{c}\text { Government/Public } \\
\text { Institution Policy } \\
\text { Brochure }\end{array}$ & 10 & 11.24 \\
\hline & Internet portal site & 26 & 29.21 \\
\hline & $\begin{array}{c}\text { SNS(Facebook, } \\
\text { Instagram, Youtube etc) }\end{array}$ & 37 & 41.57 \\
\hline & Sum & 89 & 100 \\
\hline
\end{tabular}

'2018 인터넷이용실태조사'에 따르면 모바일웹을 기반으로 한 인터넷 이용의 목적은 커뮤니케이션이 $95.2 \%$, 자료 및 정보획득이 $94 \%$ 로 나타났다. 이에 따라 본 연구는 모바일웹을 기반으로 공공데이터의 효과적 전달을 위한 시각화에 있어 중요하게 고려해야 할 요인을 연구하기 위해 스마트폰 사용자가 가장 많은 세대인 20 40 대를 
대상으로 설문조사를 실시하였고, 정보시각화의 유형별로 인지 효과를 고려한 커뮤니케이션 효과를 연구하기 위한 실험조사를 설계 및 실시하였다. 본 조사는 2018 년 11 월 내 한 달간 진행하였으며 모든 조사에 성실히 응답해준 12 명의 답변을 대상으로 분석하였다. 참여대상의 인구 통계학적 특성은 다음과 같다.

\section{2. 도구 및 평가 척도}

Table 4: General characteristics of survey subjects

\begin{tabular}{|c|c|c|}
\hline $\begin{array}{c}\text { Evaluation } \\
\text { factor }\end{array}$ & $\begin{array}{l}\text { Effects of information } \\
\text { visualization }\end{array}$ & Definition \\
\hline Clarity & $\begin{array}{l}\text { Is it clearly displayed } \\
\text { for easy recognition by } \\
\text { the user when providing } \\
\text { a lot of data at the same } \\
\text { time? }\end{array}$ & $\begin{array}{l}\text { Is it easy to recognize } \\
\text { the variety of information } \\
\text { shown in infographics? }\end{array}$ \\
\hline $\begin{array}{l}\text { Screen } \\
\text { Layout }\end{array}$ & $\begin{array}{l}\text { Is the composition of } \\
\text { the screen a visual } \\
\text { structure that is easy to } \\
\text { read and easy to } \\
\text { identify? }\end{array}$ & $\begin{array}{l}\text { Is the organization of } \\
\text { infographics arranged so } \\
\text { that information can be } \\
\text { easily understood? }\end{array}$ \\
\hline $\begin{array}{c}\text { Variable } \\
\text { recognition }\end{array}$ & $\begin{array}{l}\text { Does the relationship } \\
\text { and difference of } \\
\text { information clearly show? }\end{array}$ & $\begin{array}{l}\text { Do you clearly show } \\
\text { the relationship and } \\
\text { differences between } \\
\text { infographics' } \\
\text { information? }\end{array}$ \\
\hline Contents & $\begin{array}{l}\text { Is the content provided } \\
\text { useful? }\end{array}$ & $\begin{array}{l}\text { Is the information } \\
\text { provided by Infographics } \\
\text { useful? }\end{array}$ \\
\hline Interest & $\begin{array}{l}\text { Can the information } \\
\text { provided impart emotion } \\
\text { to the viewer's interest? }\end{array}$ & $\begin{array}{l}\text { Does the elements } \\
\text { provided in infographics } \\
\text { (images, texts, etc.) help } \\
\text { stimulate interest? }\end{array}$ \\
\hline Association & $\begin{array}{l}\text { Can you clearly } \\
\text { recognize the correlation } \\
\text { of information? }\end{array}$ & $\begin{array}{lr}\text { Can infographics } \\
\text { clearly recognize the } \\
\text { correlation } \\
\text { information? }\end{array}$ \\
\hline Harmony & $\begin{array}{l}\text { Are visual elements } \\
\text { such as text and graphics } \\
\text { on the screen in } \\
\text { harmony? }\end{array}$ & $\begin{array}{l}\text { Are the texts (forms, } \\
\text { colors), icons and } \\
\text { images of infographics } \\
\text { applied in harmony? }\end{array}$ \\
\hline Metaphor & $\begin{array}{l}\text { Are metaphor elements } \\
\text { applied to make } \\
\text { information easier to } \\
\text { understand? }\end{array}$ & $\begin{array}{l}\text { Are metaphor } \\
\text { (metaphorical and } \\
\text { metaphorical) elements } \\
\text { applied to make it easier } \\
\text { to understand the } \\
\text { information } \\
\text { infographics? }\end{array}$ \\
\hline
\end{tabular}

본 연구에서는 Lee and Lee(2018), Lee(2017) 등 총 15편의 선행 연구를 통해 효과적인 정보전달을 위한 관점에서 인포그래픽스 관련 도구를 조사하였으며, 최종적으로 명확성, 메타포, 흥미유발 등을 포함한 총 8가지를 제안한 Chwa and
$\operatorname{Kim}(2014)$ 의 연구를 기반으로 각 요인들의 평가 척도를 재구성하였다. 우선 효과적인 정보 전달을 위한 시각화에 있어 고려되어야 할 중요한 요인들은 아래와 같으며 이를 기반으로 개별 항목들을 구성하였다.

Table 5: Explanation of questionnaire about cognitive load

\begin{tabular}{|c|c|}
\hline category & Item No. \\
\hline \multirow{4}{*}{$\begin{array}{l}\text { Physical } \\
\text { Effort }\end{array}$} & $\begin{array}{l}\text { PHY1. After learning to understand the content } \\
\text { presented in Infographics' public data, I felt tired... }\end{array}$ \\
\hline & $\begin{array}{l}\text { PHY2. I felt physically exhausted in the process of } \\
\text { understanding what was presented in Infographics' } \\
\text { public data. }\end{array}$ \\
\hline & $\begin{array}{l}\text { PHY3. I felt physically tired while learning to } \\
\text { understand the content presented in Infographics' } \\
\text { public data. }\end{array}$ \\
\hline & $\begin{array}{l}\text { PHY4. I felt physical fatigue as I read the content } \\
\text { presented in Infographics' public data. }\end{array}$ \\
\hline \multirow{4}{*}{$\begin{array}{l}\text { Mental } \\
\text { Effort }\end{array}$} & $\begin{array}{l}\text { MEN1. I tried to understand what was presented in } \\
\text { Infographics' public data }\end{array}$ \\
\hline & $\begin{array}{l}\text { MEN2. I worked hard to understand what was } \\
\text { presented in Infographics' public data. }\end{array}$ \\
\hline & $\begin{array}{l}\text { MEN3. I didn't study hard to remember what was } \\
\text { presented in Infographics' public data.(Reverse) }\end{array}$ \\
\hline & $\begin{array}{l}\text { MEN4. We did our best to understand what was } \\
\text { presented in Infographics' public data. }\end{array}$ \\
\hline \multirow{4}{*}{$\begin{array}{c}\text { Task } \\
\text { Difficulty }\end{array}$} & $\begin{array}{l}\text { DIF1. It was not easy to understand what was } \\
\text { presented in Infographics' public data. }\end{array}$ \\
\hline & $\begin{array}{l}\text { DIF2. The difficulty of the content presented in the } \\
\text { infographics' public data was high. }\end{array}$ \\
\hline & $\begin{array}{l}\text { DIF3. I had a hard time understanding the } \\
\text { information presented in the information presented } \\
\text { in Infographics' public data. }\end{array}$ \\
\hline & $\begin{array}{l}\text { DIF4. The process of understanding what was } \\
\text { presented in Infographics' public data was } \\
\text { straightforward. }\end{array}$ \\
\hline \multirow{4}{*}{$\begin{array}{c}\text { Self } \\
\text { Evaluation }\end{array}$} & $\begin{array}{l}\text { SEV1. I think you have successfully understood the } \\
\text { content presented in the infographics' public data. }\end{array}$ \\
\hline & $\begin{array}{l}\text { SEV2. It seems that the contents presented in the } \\
\text { infographics' public data can be applied } \\
\text { appropriately. }\end{array}$ \\
\hline & $\begin{array}{l}\text { SEV3. After understanding the content presented in } \\
\text { Infographics' public data, I felt satisfied. }\end{array}$ \\
\hline & $\begin{array}{l}\text { SEV4. I think you have effectively understood the } \\
\text { content presented in Infographics' public data. }\end{array}$ \\
\hline \multirow{4}{*}{$\begin{array}{l}\text { Material } \\
\text { Design }\end{array}$} & $\begin{array}{l}\text { DES1. Infographics' public data information was } \\
\text { easy to read. }\end{array}$ \\
\hline & $\begin{array}{l}\text { DES2. The organization of information in the public } \\
\text { data of Infographics was made easy to use. }\end{array}$ \\
\hline & $\begin{array}{l}\text { DES3. The information of infographics' public data is } \\
\text { structured to make it easy to understand the core of } \\
\text { the content presented. }\end{array}$ \\
\hline & $\begin{array}{l}\text { DES4. It was easy to grasp the structure of } \\
\text { information in the public data of Infographics. }\end{array}$ \\
\hline
\end{tabular}


연구 문제 중 하나인 정보시각화에 대한 인지부하를 확인하기 위해 신체적 노력, 정신적 노력, 과제의 난이도, 자기 평가, 자료 설계, 총 5 가지 범주를 기반으로 범주당 4 개씩 총 12 개의 항목을 Table 5 와 같이 재구성하였다. 각각의 본 설문은 리커트 7 점 척도를 기반으로 '전혀 아니다’에서 '매우 그렇다’까지 구분할 수 있도록 하였다.

본 연구에서는 효과적인 정보시각화를 그래픽으로서 표현한 인포그래픽스 유형을 '지도형 ', '도표형 ', '타임라인형 ', '스토리텔링형 ', '만화형 ', '비교분석형 ', 총 6 개로 구분하여 유형별 사례를 보여준 인지부하 관련 문항의 '신체적 노력', '정신적 노력', '의', '자기 평가', '자료 설계'의 5 개 항목을 공공데이터를 이해하기 위한 인지부하의 평가 요소로 설정하였다.

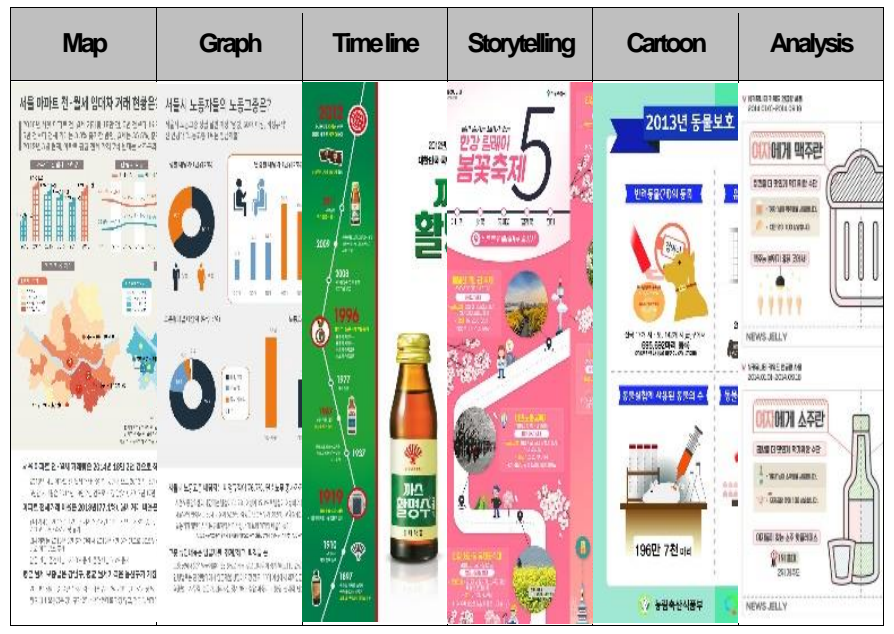

Figure. 1: Example infographics by type

PA(Importance Performance Analysis)는 고객의 만족도를 파악하기 위해 이용 전 요인들의 중요도와 이용 후 만족도를 평가하는 것으로써 상대적 비교 평가가 가능한 분석 방법이다. 본 연구에서는 참여대상에게 제공한 인포그래픽스의 사용성에 대한 평가 결과를 분석하기 위해 PA 를 실시하였다. 본 연구에서 $\mathbb{P A}$ 의 '중요도'는 향후 공공데이터의 효과적인 정보 전달을 위해 '미래'지향적 관점에서 인포그래픽스의 사용성 평가 항목에 대한 우선순위, 즉 위계의 척도로써 활용하였으며, '만족도'는 '현재'의 효과적인 정보 전달에 대한 인식을 평가하기 위한 척도로써 활용하였다. 이를 기반으로 효과적인 정보 전달을 위한 인포그래픽스의 사용성 항목들을 도출하였으며, 도출된 결과를 기반으로 $\mathrm{IPA}$ 의 분석기준에 따라 총 4 사분면의 영역에 맞추어 항목을 배치하였다. 최종적인 $\mathbb{P A}$ 모형은 1 사분면(지속가능성)은 '유지 필요 영역', 2 사분면(노력 지향)은 '사용성의 향상, 보완영역', 3 사분면(낮은 우선순위)은 '조정 영역', 4 사분면(과잉노력 지향)은 '축소 필요 영역'으로 재구성하였다.

\section{3. 분석 방법}

본 연구는 SPSS 210 패키지를 활용하여 인구통계학적 변인을 확인하기 위한 빈도 분석, 설문 항목에 대한 Cronbach Alpha 검증 및 연구 문제에 대한 최종 검증을 위해 $\mathbb{P A}$ 를 활용하여 평균 비교분석을 실시하였다. 전체 조사는 2018 년 11 월(1 개월) 내 진행되었으며 수도권 내 거주하는 20 40 대 사이의 스마트폰 보유자를 대상으로 진행하였고 그 중 성실하게 응답되어진 12 개의 설문지를 중심으로 분석하였다.

\section{4. 연구결과}

\section{1. 신뢰도 분석 결과}

설문 내용에 대한 신뢰도 분석 결과는 중요도 분석에서 지도형 0.947 , 도표형 0.954, 타임라인형 0.940, 스토리텔링형 0.951, 만화형 0.948, 비교분석형 0.951 , 만족도 분석에서 지도형 0.950, 도표형 0.949, 타임라인형 0.922, 스토리텔링형 0.951, 만화형 0.943, 비교분석형 0.942 으로 양쪽 모두 0.9 이상으로 높은 수준의 신뢰도를 보여주었다. 전체 항목에 대한 신뢰도도 지도형 0.948, 도표형 0.951, 타임라인형 0.932, 스토리텔링형 0.951 , 만화형 0.951 , 비교분석형 0.947 으로 모두 0.9 이상으로 높은 수준의 신뢰도를 나타냈다.

Table 6: Reliability analysis result

\begin{tabular}{|c|c|c|c|}
\hline $\begin{array}{c}\text { Infographics } \\
\text { types }\end{array}$ & $\begin{array}{c}\text { All } \\
\text { Cronbach } \\
\text { Alpha } \\
\text { coefficient }\end{array}$ & $\begin{array}{c}\text { Importance } \\
\text { Cronbach } \\
\text { Alpha } \\
\text { coefficient }\end{array}$ & $\begin{array}{c}\text { Satisfaction } \\
\text { Cronbach } \\
\text { Alpha } \\
\text { coefficient }\end{array}$ \\
\hline Map & 0.948 & 0.947 & 0.950 \\
\hline Chart & 0.951 & 0.954 & 0.949 \\
\hline Timeline & 0.949 & 0.940 & 0.922 \\
\hline Storytelling & 0.951 & 0.951 & 0.951 \\
\hline Animation & 0.951 & 0.948 & 0.943 \\
\hline $\begin{array}{c}\text { Comparison } \\
\text { analysis }\end{array}$ & 0.947 & 0.951 & 0.942 \\
\hline
\end{tabular}

\section{2. 공공데이터의 인포그래픽스 정보시각화 요소에 대한 전체 IPA 분석 결과}

본 연구의 $\mathbb{P A}$ 분석 결과는 전반적으로 설문결과의 중요도와 만족도의 차이 순위와 일치하는 방향성을 보였다. 공공데이터의 
인포그래픽스 정보시각화 요소에 대한 전체 $\mathbb{P A}$ 모형에 대한 분석 결과는 다음과 같다.

Table 7: All IPA analysis results

\begin{tabular}{|l|c|}
\hline \multicolumn{1}{|c|}{ Type } & Step-by-step items \\
\hline I quadrant (Sustainability) & Content, clarity \\
\hline II quadrant (Effort oriented) & Screen Layout \\
\hline II quadrant (Low priority) & $\begin{array}{c}\text { Harmony, variable perception, } \\
\text { metaphor }\end{array}$ \\
\hline $\begin{array}{l}\text { IV quadrant (Orientation of } \\
\text { excess effort) }\end{array}$ & Degree of interest \\
\hline
\end{tabular}

정보시각화의 요소 중 화면구성, 명확성, 콘텐츠, 총 3 가지는 1 사분면 지속가능 영역에 위치하며, 흥미유발의 경우 노력 지향 영역인 2 사분면의 경계면에 위치해 있다. 이는 중요도에 비해서 만족도가 낮은 것으로 즉, 인포그래픽스를 구성할 시 이미지와 텍스트를 잘 활용하여 정보를 표현하는데 보완이 필요하다는 의미로 해석되어진다.

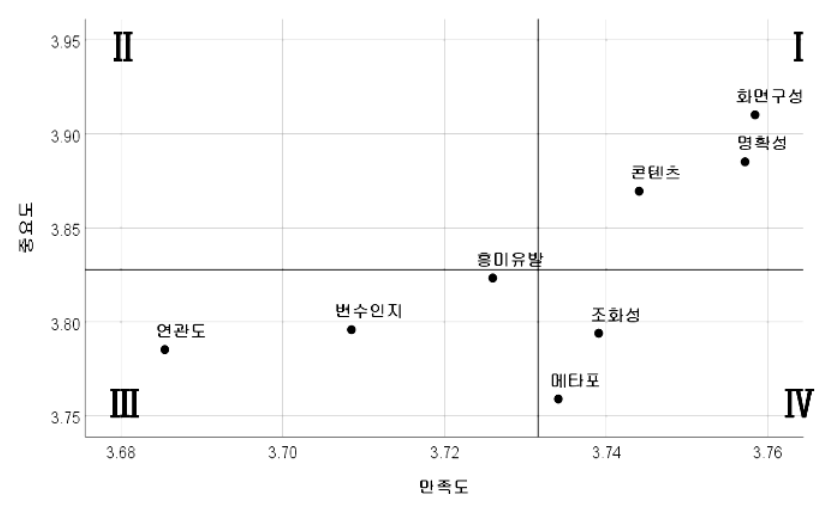

Figure 2: IPA analysis results for all information visualization elements of public data infographics

\section{3. 유형별 인포그래픽스에 대한 인지부하 조사결과}

본 연구의 공공데이터의 유형별 인포그래픽스에 대한 인지부하에 대한 분석 결과는 Table8과 같다.

Table 8: Data Information Visualization infographics

\begin{tabular}{|c|c|c|c|c|c|c|c|}
\hline Infographics types & Map & Chart & Timeline & Storytelling & Animation & Comparison analysis & Overall Average \\
\hline Physical Effort & 4.50 & 4.97 & 4.84 & 5.06 & $5.50^{*}$ & $5.19^{* *}$ & 5.01 \\
\hline Mental Effort & 5.38 & $5.47^{*}$ & 5.34 & 4.97 & 5.28 & $5.47^{*}$ & 4.69 \\
\hline Task Difficulty & 4.06 & 4.50 & 3.94 & 4.75 & $4.97^{*}$ & 5.48 \\
\hline Self Evaluation & 5.28 & 5.53 & 5.19 & $5.72^{*}$ & 5.22 & $5.72^{*}$ & 5.74 \\
\hline Material Design & 5.47 & 5.66 & $6.02^{*}$ & 5.84 & 5.47 & $5.70^{*}$ \\
\hline Overall Average & 4.94 & 5.23 & 5.07 & 5.27 & $5.29^{* *}$ & $5.37^{*}$ & 5.19 \\
\hline
\end{tabular}

인지부하 분석 결과, 총 5 가지 항목 중 첫 번째, 신체적 노력 영역에서 긍정적 결과를 나타낸 것은 인포그래픽스 유형은 만화형과 비교분석형이다. 즉, 이 2 개의 유형은 사용자가 신체적인 피곤함을 덜 느끼도록 정보를 제공하였다고 볼 수 있다. IPA 결과를 통해 만화형과 비교분석형을 살펴보면 중요도에서는 명확성, 콘텐츠, 화면구성 등의 항목이 공통적으로 높게 나타났으며, 만화형의 경우 명확성에 대한 중요도 대비 만족도는 낮게 나타났다. 따라서 만화형으로 인포그래픽스 구성 시에는 연관도와 화면구성의 보완이 필요할 것으로 보인다.

두 번째, 정신적 노력의 영역에서는 스토리텔링형이 정신적인 피로도가 가장 낮으며, 상대적으로 도표형과 비교분석형이 정신적 피로도가 높은 유형인 것으로 나타났다. 스토리텔링형의 경우 $\mathbb{P A}$
결과를 살펴보면 화면구성, 명확성, 흥미유발, 콘텐츠 등이 중요도 대비 만족도가 평균이상이거나 평균에 근접한 결과를 보이고 있어 스토리텔링형의 경우, 정보를 인지하는 데 있어 정신적 피로도와 더불어 만족도에 큰 기여를 하는 것으로 생각된다.

세 번째, 과제난이도 영역에서는 만화형이 제시된 과제를 이해하는데 비교적 용이한 것으로 나타났다. 만화형의 $\mathrm{PA}$ 결과를 살펴보면 화면구성, 콘텐츠, 흥미유발, 메타포, 명확성에 대한 중요도가 높게 나타났는데, 명확성의 경우 중요도에 비해 만족도가 낮아 향후 활용 시 보완이 필요할 것으로 보인다.

네 번째, 자기평가 영역에서는 스토리텔링형 인포그래픽스와 비교분석형 인포그래픽스가 높게 나타났다. 스토리텔링형과 
비교분석형의 $\mathbb{P A}$ 의 결과를 보면 화면구성, 명확성, 콘텐츠 등의 항목이 공통적으로 중요도가 높게 나타났으며, 스토리텔링형에서는 콘텐츠가 중요도 대비 만족도가 낮게 나타났고, 비교분석형에서는 화면구성이 중요도 대비 만족도가 낮게 나타났다. 따라서 스토리텔링형에서는 콘텐츠를 더욱 보완해 사용자를 만족할 수 있도록 해야 하고, 비교분석형에서는 화면구성에 있어 사용자 만족도를 높이기 위한 방안을 마련해야 할 것으로 보인다.

마지막, 자료설계 영역에서는 타임라인형 인포그래픽스 유형이 가장 사용자 인지에 대해 긍정적 결과를 나타내었다. 타임라인형은 화면구성, 명확성, 콘텐츠, 흥미유발 등의 주요 항목이 중요도 대비 만족도가 높은 것으로 나타났으며, 그 중 화면구성 항목은 중요도와 만족도가 가장 높게 나타났다.

\section{5. 연구결과 토론 및 시사점}

본 연구는 모바일웹 기반의 공공데이터의 효과적인 전달을 위한 정보시각화 방안을 제안하는 것이다. 세부적으로 사용자의 인지부하를 고려하여 인포그래픽스를 통해 공공데이터의 효과적 전달을 위한 정보시각화 방안을 제시하고자 하였다. 이를 위해 사례 조사 및 분석 통해 공공데이터의 인포그래픽스 유형 별 사례를 선정하고, 이를 바탕으로 효과적인 정보시각화의 표현 요소에 대해 20 30 대인 청년세대를 대상으로 중요도와 만족도 설문조사를 실시하여 그 결과에 대해 PA 를 실시하였다. 또한 20 30 대인 청년세대와 고령층 진입층인 50 60 대를 대상으로 공공데이터의 정보시각화 인지부하 실험을 실시하였다.

이러한 연구를 통해 다음의 결론을 제시한다.

첫째, 공공데이터의 효과적인 전달을 위해 정보시각화에서 명확한 데이터를 통해 식별이 용이한 화면의 구성이 우선적으로 필요하다. 적당한 그래픽 요소는 유형에 따라 활용하면 사용자가 더욱 쉽게 정보를 습득하고 이해할 수 있다. 데이터의 보유형태에 따라 다양하게 표시할 수 있을 뿐 아니라 배경의 이미지를 적절히 활용하면 효과적인 정보 전달과 사용자의 흥미를 유발할 수 있다. 각 인포그래픽의 유형 별로 살펴보면 지도형에서는 그 지역 또는 국가의 정보를 지도에 표시하고 내용을 넣어 전달하는 방식이다. 해당 지역 또는 국가의 필요한 정보를 추출해 사용자들이 원하는 유용한 콘텐츠로 구성해야 한다. 전달하려는 정보가 많을 때, 쉽게 인지하기 위해 색상으로 데이터가 집중되는 부분을 표시해주고 도표를 함께 활용하여 정보의 명확성을 높여 정보를 구성해야 한다. 도표형은 그래프와 표 등을 사용해 정보를 전달하는 방식이다. 정보의 주제를 확실히 정하여 필요한 부분만 수치를 추출하여 많은 텍스트 들이 나열되지 않도록 하고, 원형,
선형 그래프, 점그래프, 막대그래프 등 정보에 따라 유용하게 표시할 수 있는 그래프를 활용하고, 색상의 구분을 줌으로써 식별하기 쉽게 구성을 해야 한다. 또 필요하 다면 픽토그램같은 그림으로 된 언어체계를 추가해서 사용자가 쉽게 인지가 가능하도록 해야 할 필요성이 있다. 타임라인형은 역사, 진화 등 히스토리를 전개하는 양상으로 정보를 전달하는데 용이한 유형이다. 가장 중요한 것은 시간의 흐름대로 구성해야 하며, 주제의 큰 틀에 대한 이미지를 활용하여 시선을 끌어야 한다. 시간의 흐름대로 구성해야 하는 만큼 연도 등에 대해 명확하게 표시해야한다. 흐름대로 식별하기 편하도록 위에서 아래로 가는 흐름으로 사용자가 읽기 편하도록 화면구성을 할 필요성이 있다. 스토리텔링형은 어떠한 사건 또는 주제에 관련해 이야기를 들려주듯 정보를 구성하는 유형이다. 정보의 흐름대로 숫자를 표시해 표현하고, 화살표나 흘러가는 물결처럼 곡선을 이용한 막대형식의 이미지를 활용하면 사 용자가 정보를 보고 식별하기 간편하다. 또한 주제 관련하여 각 번호에 해당되는 타이틀마다 관련 이미지를 활용하면 사용자의 흥미를 이끌어 내며 더욱 시선을 끌 수 있을 것이다. 만화 형은 일상생활, 행동, 직업, 심리 등과 관련된 정보를 만화적 요소를 통해 표현하는 유형이 다. 보통 일상생활과 관련된 정보를 만화형으로 구성하는데 주제와 관련된 중점이 되는 캐릭 터와 배경 이미지 등을 활용해 구성한다. 사용자들이 많이 관심 있어 하는 분야의 정보를 추출하여 배경 색상과 텍스트의 크기를 조절하여 읽기 쉬운 화면으로 구성하고 텍스트에 색상을 더하여 포인트가 되는 부분을 식별하기 쉽게 구성해야 한다. 또한, 만화형식으로 풀어내기 때문에 만화적 요소인 캐릭터를 주제에 맞게 사용하고, 은유와 비유적 요소를 잘 활용해 정보의 이해를 도와 내용을 명확히 표시해야 할 필요성이 있다. 비교분석형은 두 개 이상의 어떤 제품 또는 개념을 비교하는 방식으로 구성된다. 비교를 하는 유형인만큼 해당 비교 대상의 상관관계가 명확한지 확인하여 정보를 구성해야 하고, 차이가 명확하게 나타나는지를 확실하게 구분이 돼야 한다. 구분을 위해 서로 다른 색상으로 차이를 주는 것이 사용자가 식별하기 좋다. 그리고 시각적으로 여러 정보를 인지하기 쉽게 하기 위해서 서로 상반되는 이미지를 활용하고 그래프와 아이콘 등을 활용하여 정보를 구성해야 할 필요성이 있다.

둘째, 공공데이터의 효과적인 전달에서 필요한 사용자의 신체적, 정신적 부담을 줄이고, 데이터의 이해도를 증가하기 위해서는 정보에 대한 흥미유발과 메타포를 형성할 수 있는 정보의 시각화가 필요하다. 즉, 정보에 대한 이해를 돕기 위해 은유 또는 비유에 대한 시각화를 통해 사용자의 흥미를 유발할 수 있어야 한다. 이를 위해 주로 활용할 수 있는 인포그래픽스를 통한 정보의 시각화 방안은 캐릭터 등의 만화적 요소를 활용하거나 일상생활과 관련된 행동, 심리 등을 활용한 정보, 두 가지 이상의 정보 유형이나 개념을 비교함으로써 사용자의 이해를 돕는 것이 
필요하다. 예를 들어 문화예술교육을 받은 아이들에 관련한 정보로 정서적, 사회적, 행동적 측면의 변화를 아이 캐릭터를 이용하여 강조하고, 상황에 따른 감정의 변화를 캐릭터의 얼굴의 표정으로 표현하며, 직관적으로 볼 수 있도록 그래프를 활용해 숫자를 표시해 줄 필요성이 있다. 또한 만화형의 예시로 정부의 정책에 관련해 예를 들면, 해당 연도의총 예산에 관련해 색상과 텍스트의 크기를 크게 하여 포인트를 주어 눈에 띄게 배치하고 계산기, 동전모양 등의 이미지를 활용하고 캐릭터가 설명하는 듯 말풍선 모양을 활용하여 설명 하듯 화면을 구상하여 배치하면 사용자에게 흥미를 주며 명확한 정보를 전달할 수 있다. 두가지 이상의 정보나 개념을 비교한 예시로 전기자동차와 가솔린자동차를 비교했을 때 전기와 가솔린하면 떠오르는 초록색 계열, 노란색 계열 등의 대표 색상을 정해주고 양 옆으로 배치를 하고 아이콘을 더하여 해당 정보를 설명하는 글을 적어준다. 색상의 구분으로 일차적으로 사용자는 비교의 대상을 한 눈에 식별할 것이고 그에 따른 아이콘 활용에 더함에 따라 정보를 이해하는데 있어서 간편하게 인지할 것이다.

셋째, 공공데이터의 효과적인 전달을 위해 정보 시각화에서 유용한 콘텐츠의 제공이 필요 하다. 공공데이터는 정부 또는 공공기관이 보유하는 있는 데이터로서 사용자의 전반적인 생활 편의성 확보와 이를 기반으로 한 정책참여 등 다양한 기능을 제공하고 있다. 따라서 교통, 기상, 의료, 경제, 환경, 여가 등에서 사용자의 일상생활에 직접적으로 관련이 될 수 있는 콘텐츠의 시각적 위계 설정과 시간적, 공간적, 분야별 변화를 나타냄으로써 현재의 상황을 용이하게 파악할 수 있는 유용한 콘텐츠 등의 제공이 필요하다. 예를 들어 공공데이터의 효과적 전달을 위한 정보 시각화의 분야를 대중교통, 자율주행차 등의 버스, 지하철의 유동인 구나 많이 이용하는 지역 등과 대중교통의 이용률, 자율주행차의 현 상황 등 나타내는 교통 분야 콘텐츠, IOT 헬스케어, 보건복지, 의료서비스 등 필수 예방주사 정보, 부정맥 예방방법, 치과의료 보험의 현황 등 의료분야에 관련된 콘텐츠, 화재, 범죄, 자연재해 등의 안전을 위한 산사태, 태풍, 산불, 지진 등의 예방법 및 대피법을 소개하고 범죄에 취약한 지역 등을 나타내는 콘텐츠, 생활 등에 관한 미세먼지 예방법 등의 안전분야, 지역 문화 및 여행 등 벚꽃놀이 주요 장소, 내일로 기차여행 지역, 가장 많이 가는 해외여행지 등을 나타내는 여가분야 콘텐츠, 주거, 교육, 정부의 경제 지원 등 어느 지역의 아파트 전월세 임대차 거래 현황, 사교육의 지역 격차, 내집 마련의 현황, 정부의 창업지원, 청년의 취업현황 등 사용자의 생활과 직간접적으로 연관이 있는 콘텐츠 등으로 구성함으로써 현재 상황과 과거의 변화 추이 등 세분화된 데이터의 제공과 더불어 유용함을 강조할 필요가 있다. 이를 위해 일상생활이나 어떠한 행동, 직업, 심리 등과 관련된 흥미성 자료를 기반으로 한 정보를 중심으로 캐릭터 등의 만화적 요소를 활용하여 정보를 전달하는 것도 신체적, 정신적 측면에서 피곤하지 않고 직관적으로 흥미성을 주는데 도움이 될 수 있을 것이다.
넷째, 공공데이터의 효과적인 전달을 위해 사용자의 이해가 용이한 내용 및 구성을 위한 정보의 시각화가 필요하다. 이를 위해 공공데이터에 대해 시간적 전개, 경로의 전개를 통해 전반적인 사항을 포괄적으로 이해할 수 있는 스토리텔링 방식의 정보시각화가 필요하다. 또한 제품 또는 개념을 두 가지 이상 비교하는 방식인 비교분석형의 정보시각화가 필요하다.

본 연구는 궁극적으로 정부 및 공공기관에서 제시하는 정부 정책 관련 공공데이터에 대해 사용자의 원활한 인식과 이해를 목표로 인포그래픽스 등에 관한 정보시각화의 기초 자료 제공에 있다. 이에 연구 결과를 통해 도출된 문제점과 개선점을 고려하여 정보시각화에 적용한다면 사용자들이 스마트폰을 통해 정보를 습득 시 효과적인 정보 전달이 가능해질 것이다. 이러한 결과에도 불구하고 본 연구는 다음과 같은 한계점을 지니고 있다. 첫째, 성별, 거주지역의 차이에 따라 시각화된 정보의 인지 차이가 있을 수 있다. 따라서 향후 연구대상을 세분화하여 공공데이터의 사용자를 대표할 수 있는 대상을 모집하여 구체적 시각화된 공공데이터 정보의 효과적인 방법에 대한 연구할 필요가 있다. 둘째, 본 연구를 진행하며 물리적 한계로 인하여 더 많은 사용자를 조사를 할 수 없었던 부분도 연구의 한계로 남는다. 이와 같은 한계점을 극복하기 위해 다양한 측정 방법을 연구하고 개발하는 것이 필수적이며 더 많은 논의를 통해 공공데이터의 정보시각화와 관련된 다양한 연구가 필요하다. 셋째, 본 연구는 20 40 대의 젊은층을 대상으로 연구를 진행하였다. 공공데이터의 특성상 전국민을 대상으로 하므로 샘플의 선정을 다양한 연령별로 선정했어야 하나 20 40 대의 젊은 세대를 위주로 선정된 점은 본 연구의 한계점이다. 특히 우리나라와 같이 노령화가 빠르게 진행되는 나라에서는 디지털 약자를 위한 공공데이터의 정보시각화에 대한 추가적인 연구가 필요하다.

\section{References}

Ahn, C. M. (2016). A Study on the current status of Data Visualization. Proceedings of Symposium of the Korean Institute of communications and Information Sciences, 12, 764-765.

Cho, E. H., Kim, H. O., \& Ryoo, H. Y. (2011). Image-based Visualization of Realtime Numeric Data on a Smartphone For Designing a Water Quality Measurement Application. Journal of Digital Design, 11(4), 91-100.

Choi, J. H., Kim, D. S., \& Kim, H. W. (2014). An Audit Model for Usability of the Mobile Web UI/UX. Journal of Korea Knowledge and Technology, 11(2), 163-175.

Choi, J. W., \& Kim, L. Y. (2012). A Study on Infographic for Effective Visual Communication of the Big Data Era Government Departments and Public Institutions-. Korea Science \& Art Forum, 11(10), 94-103. 
Chung, K. J., \& Lee, B. K. (2016). Comparison of usage patterns of mobile web and app and ecosystem of mobile internet service. KISDI Research Paper, 16(3), 12-21.

Chwa, E. H., \& Kim, H. H. (2014). A Study on the Effective Communication of Information Visualization - Focusing on the First Page of Weather Forecasting Application. Digital Design Studies, 14(3), 83-94.

Doo, K. I. (2016). A Study on Infographic for Effective Visualization of Big Data. Journal of Communication Design, $55,152-161$.

Ikehara, C. S., \& Crosby, M. E. (2005). Assessing Cognitive Load with Physiological Sensors. Proceedings of the 38th Hawaii International Conference on System Sciences, 1, 1-5. ISBN 07695-2268-8.

Jin, J. A. (2018). A Study on Infographics Research Trend from 2004 to 2017: Focusing on the Content Analysis of Academic Journal Articles in Korea. Korea Design Forum, 23(1), 135146.

Kim, B. T., \& Kim, Y. H. (2010). A study on user interface designs through analysis of information visualization in a mobileaugmented reality. Journal of Korea Design Knowledge, 16, 72-81.

Kim, B. N., Choi, Y. H., \& Park, S. H. (2014). A Study on Interactive Visualization Guideline of Spatial-temporal Information -Based on Use Context of Location Based Social Network Service-. Design Convergence Service, 13(6), 39-56.

Kim, M. J., \& Chung, H. J. (2014). A Case Study on Visual Expression through Interaction with Information Types Focusing on Interactive Infographic in the New York Times-. Korea Society of Design Culture, 20(1), 145-158.

Kim, J. E., Kim, W. J., Leem, K. W., \& Sohn, Y. S. (2018). A Study on the data analysis platform for effective analysis of public data. Proceedings of Symposium of the Korean Institute of communications and Information Sciences, 21, 313-314.

Kim, J. I. (2018). A proposal for the direction of information visualization to revitalize the national public data portal service (Master's Thesis). Hongik University, Seoul, Korea.

Kim, T. H., Park, B. J., \& Suh, E. K. (2019). Irregular Bigdata Analysis and Considerations for Civil Complaint Based on Design Thinking. Journal of Industrial Distribution \& Business, 9(8), 51-60.

Lee. G. D. (2018). Research on design thinking in the use of infographic visual elements. Korean Society for Product Culture and Design, 54(10), 89-99.

Lee, H. N. (2017). Analysis of Characteristics Through Information Visualization Stereotyping - The Case of a Special Emphasis on Social Curation SNS Cases -. Journal of the Korean Society Design Culture, 23(2), 547-556.

Lee, J. M. (2017). The Influence of Visual Elements and
Interaction on Understanding Information Visualization (Master's Thesis). Seoul National University, Seoul, Korea.

Lee, J. S. (2013). A Study on Visualizing Method and Expression of Information Design for Big Data. Bulletin of Korean Society of Basic Design \& Art, 14(3), 261-269.

Lee, S. Y., \& Lee, H. S. (2020). A Study on the Smart Tourism Awareness through Bigdata Analysis. Journal of Industrial Distribution \& Business, 11(3), 19-28.

Lip Source Korea. (2018). Analysis of the results of the 2nd survey of government departments' work / promotion. Retrieved September 11, 2019, from https://blog.naver.com/ipsos_korea/221416920070.

Oh. B. K., \& Kang, S. C. (2008). Textbook of information design (1st ed.). Paju, Korea: Ahngraphics.

Park, J. C., Choi, Y. L, \& Kim, I. G. (2020). Analysis of Taekwondo Performances Using IPA Technique. Sport Science, 37(2), 95-104.

Park, S. H. (2018). A study on the effect of service quality on business performance of the roastery coffee shops as a differentiation factor (Master's Thesis). Chung-Ang University, Seoul, Korea.

Peak15 Communications. (2012). Communicate with Data! 6 types of Infographics. Retrieved September 06, 2019, from https://peak15.tistory.com/330.

Ryu, J. H., \& Yim, J. H. (2009). An Exploratory Validation for the Constructs of Cognitive Load. The Journal of Educational Information and Media, 15(2), 1-27.

Shin, H. S., Leem, J. M., \& Park, J. S. (2013). Information Visualization and Information Presentation for Visually Impaired People. Electronics and telecommunications trends, 28(1), 81-91.

Song, S. J. (2011). A Study of GUI design about button's size and Location on the mobile Web (Master's Thesis). Dongseo University, Busan, Korea.

Yoo, D. H. (2016). The effect of visual embellishment on user information processing type in the information visualization (Master's Thesis). Hongik University, Seoul, Korea.

Yoo, H. S., Suh, E. K., \& Kim, T. H. (2020). A Study on Technology Acceptance of Elderly living Alone in Smart City Environment: Based on AI Speaker. Journal of Industrial Distribution \& Business, 11(2), 41-48.

Yoo, I. H. (2017). Type Analysis of Visual Expressions based on Infographic Used by Government Branches - Focusing on Work Reports of 25 Government Branches. Journal of Communication Design, 60, 173-188.

Zuo, Y. X., \& Kim, H. H. (2014). A Study on the Effective Communication of Information Visualization - Focusing on the First Page of Weather Forecasting Application. Journal of Digital Design, 14(3), 83-94. 